\title{
Hydromagnetic stability of differentially rotating neutron stars
}

\author{
V. Urpin ${ }^{\star}$
}

\author{
A. F. Ioffe Institute for Physics and Technology, SU 194021, St. Petersburg, Russia \\ Isaak Newton Institute of Chili, Branch in St. Petersburg, Russia
}

Received 31 March 2003 / Accepted 21 August 2003

\begin{abstract}
The stability properties of differentially rotating magnetic neutron stars are considered, and the instability criteria are obtained. The influence of the magnetic field is twofold: it may stabilize a fluid against some instabilities, on the one hand, and it can lead to new branches of instabilities, on the other hand. It turns out that some of the instability criteria of magnetic neutron stars can be satisfied at smaller departures from the uniform rotation than the criteria of non-magnetic stars. Interaction of hydrodynamic motions caused by instabilities in the core with the neutron star crust can result in small irregularities in the measured spin period of pulsars.
\end{abstract}

Key words. magnetohydrodynamics (MHD) - stars: neutron - stars: rotation - stars: pulsars: general

\section{Introduction}

Likely, young neutron stars formed in core collapse should be rapidly and differentially rotating due to the conservation of the angular momentum of the collapsing core. Recent numerical simulations of rotational core collapse indicate clearly that the remnant will indeed rotate differentially (see Zwerger \& Müller 1997; Rampp et al. 1998; Dimmelmeier et al. 2002). Soon after collapse, the neutron star undergoes various hydrodynamic instabilities (see, e.g., Miralles et al. 2000, 2002) caused by the temperature and lepton number gradients. During this stage that lasts $\sim 30-40 \mathrm{~s}$, the angular momentum transport is basically determined by convection and neutron fingers instability, and the original rotation law may evolve to even a more complex one.

Differential rotation can be the key issue not only for young neutron stars formed in core collapse but also for stars originating in the merger of a binary neutron star system. Observations of binary pulsars suggest that the mass of neutron stars in such systems is close to the canonical mass $1.4 M_{\odot}$ (Thorsen et al. 1993). The remnant of a merger will then have a mass of the order of $3 M_{\odot}$ that is larger than the maximum allowed mass for neutron stars. Therefore, the merger can lead to rapid collapse to a black hole if there is no mechanism supporting such a massive neutron star against collapse. It was pointed out by Baumgarte et al. (2000), however, that differentially rotating neutron stars can have a substantially larger rest mass than their uniformly rotating counterparts. Generally, even modest differential rotation may easily support $\geq 3 M_{\odot}$, the expected mass of merger remnants. Numerical simulations (see, e.g., Rasio \& Shapiro 1999; Shibata \& Uryu 2000) show that coalescence will indeed form a differentially rotating remnant with the core

* e-mail: vadim.urpin@uv.es rotating faster than the envelope and, hence, such remnants can likely support a large mass, at least temporary. Hydromagnetic instabilities in the remnant can destroy differential rotation and lead to delayed collapse and a delayed gravitational wave burst.

Differential rotation in neutron stars can also be induced by the instability of $r$-modes and emission of gravitational waves. For instance, very weakly magnetized accreting neutron stars in $\mathrm{X}$-ray binaries can cross the $r$-mode instability boundary when the spin period reaches $\sim 1-2 \mathrm{~ms}$ (Spruit 1999). Angular momentum loss by the gravitational waves causes strong differential rotation that can amplify the magnetic field of such neutron stars. Note, however, that even a relatively weak magnetic field $\sim 10^{10} \mathrm{G}$ can prevent gravitational radiation from exciting $r$-mode oscillations or can damp them on a short time scale (Rezzolla et al. 2000).

In this paper, we consider the stability criteria of differentially rotating hot neutron stars in the presence of the magnetic field. Our analysis is restricted to the linear stability properties. Note that apart from linear instabilities the neutron stars can also be subject to non-linear instabilities. These instabilities require a sufficiently large initial perturbations of the star and are beyond the scope of the present paper. The linear stability properties of non-magnetic neutron stars have been considered by Urpin (2003). The magnetic field can be generated in the core, for example, by turbulent dynamo mechanism during the convective stage (see, e.g., Thompson \& Duncan 1993). The field can also be amplified from a weak field of the progenitor because of the conservation of the magnetic flux (Woltjer 1964; Shapiro \& Teukolsky 1983).

Most likely, the distribution of the angular momentum in young neutron stars is rather complex in the presence of the magnetic field. Therefore, such stars can be subject to various hydromagnetic instabilities. These instabilities may generally 
differ from the instabilities arising in radiative zones of ordinary stars. In the presence of the magnetic field, the main source of turbulization in stellar radiative zones is probably the magnetic shear instability first considered by Velikhov (1959) and Chandrasekhar (1960) and analyzed in detail for stellar conditions by a number of authors (see, e.g. Fricke 1969; Acheson 1978, 1979; Balbus 1995; Urpin 1996; Kitchatinov \& Rüdiger 1997). This instability arises if the angular velocity decreases from the pole to the equator. The number of rotational instabilities in magnetic neutron stars can be larger, and the criteria of these instabilities can be different because of a more pronounced influence of kinetic processes (viscosity, thermal diffusivity).

Note that, apart from hydromagnetic instabilities, the angular momentum in magnetic neutron stars can also be redistributed by a magnetic braking mechanism (Shapiro 2000) if the magnetic field is sufficiently strong. Differential rotation twists up lines of a poloidal magnetic field and amplifies the toroidal field. This process generates Alfvén waves, which transport the angular momentum within the star and carry out some angular momentum from the star to the surrounding plasma. The efficiency of this mechanism, however, is very sensitive to assumptions regarding the density of external plasma. If this density is small then differential rotation dissipates on a viscous timescale.

The paper is organized as follows. In Sect. 2, we derive the dispersion equation governing the rotational modes of a differentially rotating magnetic neutron star. In Sects. 3, the stability criteria of magnetic neutron stars are derived. A discussion of the results is represented in Sect. 4.

\section{Rotational modes in magnetic neutron stars}

Consider the axisymmetric core of a magnetic neutron star rotating with the angular velocity dependent on both $s$ - and $z$-coordinates, so $\Omega=\Omega(s, z) ;(s, \varphi, z)$ are cylindrical coordinates. We assume the magnetic field, $\boldsymbol{B}=\left(B_{s}, B_{\varphi}, B_{z}\right)$, to be weak in the sense that the Alfvén speed, $c_{\mathrm{A}}$, is small compared to the sound speed, $c_{\mathrm{s}}$. In this case, the hydromagnetic stability can be treated by making use of the Boussinesq approximation that provides reliable results if the growth time of instabilities is longer than the period of sound waves.

We assume that, in the unperturbed state, the star is in hydrostatic equilibrium, and

$\frac{\nabla p}{\rho}=\boldsymbol{g}+\Omega^{2} \boldsymbol{s}+\frac{1}{4 \pi \rho}(\nabla \times \boldsymbol{B}) \times \boldsymbol{B}$,

where $p$ and $\rho$ are the pressure and density, respectively, and $\boldsymbol{g}$ is the gravity. If $c_{\mathrm{s}}>c_{\mathrm{A}}$, the Lorentz force is small compared to the pressure force.

Small linear perturbations are governed by the linearized magnetohydrodynamic equations. In this paper, we consider stability to axisymmetric short lengthscale perturbations with the lengthscale $\lambda \ll r=\sqrt{s^{2}+z^{2}}$. Small perturbations will be indicated by subscript 1 , whilst unperturbed quantities will have no subscript. Then, the linearized magnetohydrodynamic equations in the Boussinesq approximation read in the lowest order in $\lambda / r$

$$
\begin{aligned}
& \dot{\boldsymbol{V}}_{1}+2 \boldsymbol{\Omega} \times \boldsymbol{V}_{1}+\boldsymbol{e}_{\varphi} s\left(\boldsymbol{V}_{1} \cdot \nabla \Omega\right) \approx \\
& \quad-\frac{\nabla p_{1}}{\rho}-\beta \boldsymbol{G} T_{1}+\frac{1}{4 \pi \rho}\left(\nabla \times \boldsymbol{B}_{1}\right) \times \boldsymbol{B}+v \Delta \boldsymbol{V}_{1} \\
& \dot{\boldsymbol{B}}_{1} \approx(\boldsymbol{B} \cdot \nabla) \boldsymbol{V}_{1}+s \boldsymbol{e}_{\varphi}\left(\boldsymbol{B}_{1} \cdot \nabla \Omega\right) \\
& \nabla \cdot \boldsymbol{V}_{1} \approx 0 \\
& \nabla \cdot \boldsymbol{B}_{1}=0 \\
& \dot{T}_{1}+\boldsymbol{V}_{1} \cdot \boldsymbol{D} \approx \chi \Delta T_{1}-\varepsilon_{1} / \rho c_{\mathrm{p}}
\end{aligned}
$$

where $\boldsymbol{V}_{1}, \boldsymbol{B}_{1}, p_{1}$ and $T_{1}$ are perturbations of the hydrodynamic velocity, magnetic field, pressure and temperature, respectively; $\boldsymbol{G}=\boldsymbol{g}+\Omega^{2} \boldsymbol{s} ; \varepsilon_{1}$ is the perturbation of the neutrino emissivity; $\beta=-(\partial \ln \rho / \partial T)_{\mathrm{p}}$ is the thermal expansion coefficient; $v$ and $\chi$ are the kinematic viscosity and thermal diffusivity, respectively; $c_{\mathrm{p}}$ is the specific heat at constant pressure; $\boldsymbol{D}=\nabla T-\nabla_{\mathrm{ad}} T$ is the difference between the actual and adiabatic temperature gradients; we denote by $\boldsymbol{e}_{\varphi}$ the unit vector in the azimuthal direction.

Equation (2) is a linearized momentum equation for rotating fluid in the presence of the magnetic field (see, e.g., Chandrasekhar 1961). In this equation, the density perturbation in the buoyancy force, $\rho_{1} \nabla p / \rho^{2}$, is expressed in terms of the temperature perturbation, thus $\rho_{1}=-\rho \beta T_{1}$, in accordance with the main idea of the Boussinesq approximation. The unperturbed Lorentz force is neglected compared to the unperturbed gravity and centrifugal force in the expression for $\nabla p$ since $c_{\mathrm{s}} \gg c_{\mathrm{A}}$. In the perturbed Lorentz force, we neglect the term $\propto(\nabla \times \boldsymbol{B}) \times \boldsymbol{B}_{1}$ compared to $\left(\nabla \times \boldsymbol{B}_{1}\right) \times \boldsymbol{B}$ since we adopt a short wavelength approximation. We take into account viscosity in Eq. (2) because its timescale is generally comparable to the thermal timescale.

In the induction Eq. (3), the magnetic field is assumed to be "frozen" into the core plasma and dissipative effects are neglected. The electrical conductivity of hot nuclear matter is indeed very high, $\sim 1.5 \times 10^{26} T_{9}^{-2} \mathrm{~s}^{-1}$ where $T_{9}=T / 10^{9} \mathrm{~K}$ (Baym et al. 1971), and the characteristic timescale of ohmic dissipation is very long $\left(\sim 10^{5}-10^{6} \mathrm{yrs}\right.$ for perturbations with the lengthscale of the order of the pressure lengthscale). Therefore, the induction Eq. (3) describes the evolution of the magnetic field caused only by perturbed advection and stretching of the perturbed field lines due to unperturbed differential rotation.

In the continuity Eq. (4), we neglect the term proportional to $\dot{\rho}_{1}$ that is small in the Boussinesq approximation. We also take into account that the term $\boldsymbol{V}_{1} \cdot \nabla \rho$ is small compared to $\rho \nabla \cdot V_{1}$ for short lengthscale perturbations. The effect of thermal diffusivity is included in the standard thermal balance Eq. (6) (see, e.g., Landau \& Lifshitz 1981) since this effect can be significant for perturbations with very short wavelength. We neglect, however, viscous dissipation in Eq. (6) since its contribution is much smaller than advection of heat. Note the absence of terms proportional to $p_{1}$ on the left hand side of Eq. (6) because these terms are negligible in the Boussinesq approximation. 
The neutrino emissivity can generally be represented as a function of $\rho$ and $T, \varepsilon=\varepsilon(\rho, T)$ (see, e.g, Maxwell 1979). Then,

$\varepsilon_{1}=\frac{\partial \varepsilon}{\partial T} T_{1}+\frac{\partial \varepsilon}{\partial \rho} \rho_{1}=\left(\frac{\partial \varepsilon}{\partial T}-\beta \rho \frac{\partial \varepsilon}{\partial \rho}\right) T_{1}$.

We consider the behaviour of axisymmetric short wavelength perturbations with the space-time dependence $\exp (\gamma t-\mathrm{i} \boldsymbol{k} \cdot \boldsymbol{r})$ where $\boldsymbol{k}=\left(k_{s}, 0, k_{z}\right)$ is the wavevector, $|\boldsymbol{k} \cdot \boldsymbol{r}| \gg 1$. The dispersion equation for such perturbations reads

$\gamma^{5}+a_{4} \gamma^{4}+a_{3} \gamma^{3}+a_{2} \gamma^{2}+a_{1} \gamma+a_{0}=0$,

where

$a_{4}=\omega_{\mathrm{T}}+2 \omega_{v}, \quad a_{3}=2 \omega_{\mathrm{A}}^{2}+\omega_{\mathrm{g}}^{2}+Q^{2}+\omega_{v}\left(\omega_{v}+2 \omega_{\mathrm{T}}\right)$,

$a_{2}=\omega_{\mathrm{T}}\left(2 \omega_{\mathrm{A}}^{2}+Q^{2}+\omega_{v}^{2}\right)+\omega_{\nu}\left(2 \omega_{\mathrm{A}}^{2}+\omega_{\mathrm{g}}^{2}\right)$,

$a_{1}=\omega_{\mathrm{A}}^{2}\left(\omega_{\mathrm{A}}^{2}+\omega_{\mathrm{g}}^{2}+Q^{2}-4 \Omega^{2} \frac{k_{z}^{2}}{k^{2}}+2 \omega_{\nu} \omega_{\mathrm{T}}\right)$,

$a_{0}=\omega_{\mathrm{T}} \omega_{\mathrm{A}}^{2}\left(\omega_{\mathrm{A}}^{2}+Q^{2}-4 \Omega^{2} \frac{k_{z}^{2}}{k^{2}}\right)$,

and

$Q^{2}=4 \Omega^{2} \frac{k_{z}^{2}}{k^{2}}+2 \Omega s \frac{k_{z}}{k^{2}}\left(k_{z} \frac{\partial \Omega}{\partial s}-k_{s} \frac{\partial \Omega}{\partial z}\right)$,

$\omega_{\mathrm{g}}^{2}=-\beta(\Delta \nabla T) \cdot\left[\boldsymbol{G}-\frac{\boldsymbol{k}}{k^{2}}(\boldsymbol{k} \cdot \boldsymbol{G})\right], \quad \omega_{v}=v k^{2}$,

$\omega_{\mathrm{T}}=\omega_{\chi}+\omega_{\varepsilon}, \quad \omega_{\chi}=\chi k^{2}, \quad \omega_{\varepsilon}=\frac{1}{\rho c_{\mathrm{p}}}\left(\frac{\partial \varepsilon}{\partial T}-\beta \rho \frac{\partial \varepsilon}{\partial \rho}\right)$,

$\omega_{\mathrm{g}}$ is the frequency of buoyancy waves; $\omega_{\mathrm{A}}=(\boldsymbol{k} \cdot \boldsymbol{B}) / \sqrt{4 \pi \rho}$ is the Alfvén frequency; $\omega_{\chi}$ and $\omega_{v}$ are the inverse timescales of dissipation due to the thermal conductivity and viscosity, respectively; $\omega_{\varepsilon}$ characterizes the cooling rate due to neutrino emission.

\section{The criteria of instability of magnetic neutron stars}

Equation (8) describes five low-frequency modes which can exist in rotating magnetic neutron star. The condition that at least one of the roots of Eq. (8) has a positive real part (unstable mode) is equivalent to one of the following inequalities

$a_{4}<0, \quad a_{0}<0$,

$A_{1} \equiv a_{4} a_{3}-a_{2}<0$,

$A_{2} \equiv a_{2}\left(a_{4} a_{3}-a_{2}\right)-a_{4}\left(a_{4} a_{1}-a_{0}\right)<0$,

$A_{3} \equiv\left(a_{4} a_{1}-a_{0}\right)\left[a_{2}\left(a_{4} a_{3}-a_{2}\right)-a_{4}\left(a_{4} a_{1}-a_{0}\right)\right]$

$$
-a_{0}\left(a_{4} a_{3}-a_{2}\right)^{2}<0
$$

being fulfilled (see, e.g., Aleksandrov et al. 1985). The first condition, $a_{4}<0$, cannot be satisfied since $\omega_{\mathrm{T}}>0$ and $\omega_{v}>0$, therefore, only the four other conditions determine the instability.

In young neutron stars, we have typically $\omega_{v} \sim \omega_{\chi} \gg \omega_{\varepsilon}$. Since $\omega_{v} \propto \omega_{\chi} \propto k^{2}$ dissipative terms in Eqs. (9) increase rapidly when the wavelength decreases, and neither of the inequalities (9) can be satisfied for very short wavelengths. Therefore, perturbations with very short wavelengths are always stable, and instability can arise only for perturbations with $k$ satisfying the condition

$\min \left(\left|\omega_{\mathrm{g}}\right|,|Q|\right) \gg \omega_{v} \sim \omega_{\chi} \gg \omega_{\varepsilon}$.

Using the expression for $v$ given by Cutler et al. (1990) and taking into account that $\omega_{\mathrm{g}} \geq|Q| \sim \Omega$, we obtain from Eq. (10) the following inequality for a wavelength, $\lambda=2 \pi / k$,

$\lambda \gg \lambda_{\mathrm{c}}=33 \frac{\rho_{14}^{5 / 8}}{T_{9}} P^{1 / 2} \mathrm{~cm}$,

where $\rho_{14}=\rho / 10^{14} \mathrm{~g} / \mathrm{cm}^{3}, T_{9}=T / 10^{9} \mathrm{~K}$, and $P=2 \pi / \Omega$ is the spin period (in seconds). Only such perturbations will be treated in this paper.

\section{The condition $a_{0}<0$}

The second inequality (9) describes the criterion of the magnetorotational instability and reads

$Q^{2}-4 \Omega^{2} \frac{k_{z}^{2}}{k^{2}}+\omega_{\mathrm{A}}^{2}<0$.

We can rewrite this equation as

$s \frac{k_{z}}{k^{2}}\left(k_{z} \frac{\partial \Omega^{2}}{\partial s}-k_{s} \frac{\partial \Omega^{2}}{\partial z}\right)<-\omega_{\mathrm{A}}^{2}$.

Both the radial and vertical gradients of the angular velocity can contribute to instability. Note that the condition $a_{0}<0$ appears only due to taking into account the thermal diffusivity and neutrino emissivity. It does not appear if one considers the instability in adiabatic approximation $\left(\omega_{\mathrm{T}} \rightarrow 0\right)$ since $a_{0}$ is proportional to $\omega_{\mathrm{T}}$. In adiabatic limit, the criterion of magnetorotational instability contains an additional term, $\omega_{\mathrm{g}}^{2}$, on the left hand side that provides a strong stabilizing effect if stratification is stable. An efficient heat exchange between perturbations and surrounding plasma decreases the stabilizing influence of bouyancy in neutron stars compared to ordinary stars. The magnetic field in Eqs. (12) and (13) plays a stabilizing role and, generally, a sufficiently strong magnetic field can completely suppress the instability. From Eq. (13), we can estimate the stabilizing field, $B_{\text {st }}$, as

$B_{\mathrm{st}} \sim \frac{1}{k} \sqrt{4 \pi \rho s \mid \nabla \Omega^{2}}$.

Estimating $\left|\nabla \Omega^{2}\right|$ as $2 \Omega \Delta \Omega / s$ where $\Delta \Omega$ is the departure from a uniform rotation, we have

$B_{\mathrm{st}} \sim \frac{1}{k} \sqrt{8 \pi \rho \Omega \Delta \Omega}$.

For $\Omega \sim \Delta \Omega \sim 10^{3}$, the stabilizing field is $B_{\mathrm{st}} \sim 10^{15} \lambda_{5} \mathrm{G}$, where $\lambda_{5}=\lambda / 10^{5} \mathrm{~cm}$. This field is much stronger than the standard pulsar field, $\sim 10^{12}-10^{13} \mathrm{G}$, if $\lambda_{5} \sim 1$. For such $\lambda_{5}$, the pulsar field can suppress only a very weak departure from a uniform rotation with

$\frac{\Delta \Omega}{\Omega}<2.5 B_{13}^{2} P^{2} \lambda_{5}^{-2}$, 
where $B_{13}=B / 10^{13} \mathrm{G}$. Therefore, the stabilizing influence of the magnetic field is probably relatively weak in typical neutron stars except perturbations with a very short wavelength. For example, the standard field can be sufficient to suppress the instability of perturbations with $\lambda \leq 10 \mathrm{~m}$ if $\Delta \Omega \sim \Omega$.

To satisfy the inequality (13), the necessary condition is

$\frac{k_{z}}{k^{2}}\left(k_{z} \frac{\partial \Omega^{2}}{\partial s}-k_{s} \frac{\partial \Omega^{2}}{\partial z}\right)<0$.

For the particular case of the angular velocity being constant on cylinders, this condition can be satisfied only if $\partial \Omega / \partial s<0$. If $\partial \Omega / \partial z \neq 0$ then, for any dependence of $\Omega$ on $s$ and $z$, there exists a region in the plane $\left(k_{s}, k_{z}\right)$ where the condition (17) is fulfilled. The components of a wavevector should satisfy the inequality

$\left|\frac{k_{s}}{k_{z}}\right|>\frac{|\partial \Omega / \partial s|}{|\partial \Omega / \partial z|}$

and $k_{s}$ and $k_{z}$ are of the same/opposite sign if the quantity $(\partial \Omega / \partial s) /(\partial \Omega / \partial z)$ is positive/negative.

\section{The condition $A_{1}<0$}

Substituting the values of coefficients $a_{4}, a_{3}$ and $a_{2}$ and assuming $\lambda \gg \lambda_{\mathrm{c}}$, we can rewrite the third inequality (9) as

$\left(\omega_{\chi}+\omega_{\nu}\right) \omega_{\mathrm{g}}^{2}+2 \omega_{v}\left(Q^{2}+\omega_{\mathrm{A}}^{2}\right)<0$.

This inequality generalizes the condition of convective instability. In the case of inviscid fluid with $g \| \Delta \nabla T$, Eq. (19) reduces to the well-known Schwarzschild criterion of convection,

$\omega_{\mathrm{g}}^{2}<0$.

We can transform Eq. (19) into

$s \frac{k_{z}}{k^{2}}\left(k_{z} \frac{\partial \Omega^{2}}{\partial s}-k_{s} \frac{\partial \Omega^{2}}{\partial z}\right)+\omega_{\mathrm{A}}^{2}+4 \Omega^{2} \frac{k_{z}^{2}}{k^{2}}+\frac{\mu^{2}}{2} \omega_{\mathrm{g}}^{2}<0$,

where $\mu^{2}=1+\chi / v$. The magnetic field plays a stabilizing role in this condition as usual. However, a stabilizing influence is also provided by a stable stratification and by rotation itself. Compared to the criterion (13), the condition (21) is harder to fulfil since the both additional terms on the right hand side are positive. The necessary (but not sufficient) condition of instability represented by the inequality (21) is

$Q^{2}<0$

or

$\Omega_{\mathrm{e}}^{2} \frac{k_{z}^{2}}{k^{2}}-\frac{k_{z} k_{s}}{k^{2}} s \frac{\partial \Omega^{2}}{\partial z}<0$,

where

$\Omega_{\mathrm{e}}^{2}=\frac{\partial}{s^{3} \partial s}\left(s^{4} \Omega^{2}\right)$.

If the Rayleigh stability criterion, $\Omega_{\mathrm{e}}^{2}>0$, is fulfilled, the condition (23) (and, hence, the condition (21)) applies only if $\partial \Omega / \partial z \neq 0$.
Consider the sufficient condition of instability (21). This condition depends on the direction of $\boldsymbol{k}$. If the centrifugal force is weak compared to the gravity, $g \gg \Omega^{2} s$, then $\Delta \nabla T$ and $g$ are approximately radial, and we have

$\omega_{\mathrm{g}}^{2} \approx \frac{k_{\theta}^{2}}{k^{2}} \omega_{\mathrm{BV}}^{2}$,

where $k_{\theta}$ is the $\theta$-component of a wavevector, $k_{\theta}^{2}=k_{s}^{2} \cos ^{2} \theta-$ $2 k_{s} k_{z} \cos \theta \sin \theta+k_{z}^{2} \sin ^{2} \theta, \theta$ is the polar angle; $\omega_{\mathrm{BV}}^{2}=-\beta(\boldsymbol{g}$. $\Delta \nabla T)$ is the Brunt-Vaisala frequency, $\omega_{\mathrm{BV}}^{2}>0$ for stable stratification.

To obtain the true criterion of instability, we have to minimize the left hand side of inequality (21) as a function of the direction of $\boldsymbol{k}$. Equation (21) can be rewritten as

$E k_{s}^{2}-C k_{z} k_{s}+F k_{z}^{2}<0$,

where

$E=\Omega_{\mathrm{e}}^{2}+\frac{1}{2} \mu^{2} \omega_{\mathrm{BV}}^{2} \sin ^{2} \theta+\omega_{\mathrm{A} z}^{2}$,

$C=s \frac{\partial \Omega^{2}}{\partial z}+\mu^{2} \omega_{\mathrm{BV}}^{2} \cos \theta \sin \theta-2 \omega_{\mathrm{A} z} \omega_{\mathrm{A} s}$,

$F=\frac{1}{2} \mu^{2} \omega_{\mathrm{BV}}^{2} \cos ^{2} \theta+\omega_{\mathrm{A} s}^{2}$,

and $\omega_{\mathrm{A} s, \mathrm{~A} z}=k B_{s, z} / \sqrt{4 \pi \rho}$. Note that the coefficients $E, C$ and $F$ depend on $k^{2}$ but do not depend on the direction of $\boldsymbol{k}$. At a given $k$, the left hand side of Eq. (26) reaches its minimum if $k_{z}=C k_{s} / 2 F$, and the minimum value is $k_{s}^{2}\left(E-C^{2} / 4 F\right)$. The minimum is negative if

$C^{2}>4 E F$.

Substituting the value of coefficients $E, C$ and $F$, we can represent the sufficient condition (30) as

$$
\begin{aligned}
\left(\frac{\partial \Omega}{\partial z}\right)^{2}> & \frac{\alpha_{1}}{s^{2}}\left(\cos ^{2} \theta \Omega_{\mathrm{e}}^{2}-2 s \sin \theta \cos \theta \Omega \frac{\partial \Omega}{\partial z}\right) \\
& +\frac{\alpha_{1}}{s^{2}}\left(\omega_{\mathrm{A} s} \sin \theta+\omega_{\mathrm{A} z} \cos \theta\right)^{2} \\
& +\frac{1}{s^{2} \Omega^{2}}\left(\Omega_{\mathrm{e}}^{2} \omega_{\mathrm{A} s}^{2}+2 \Omega s \frac{\partial \Omega}{\partial z} \omega_{\mathrm{A} s} \omega_{\mathrm{A} z}\right),
\end{aligned}
$$

where $\alpha_{1}=\left(\mu^{2} / 2\right)\left(\omega_{\mathrm{BV}} / \Omega\right)^{2}$.

If the magnetic field is weak in the sense $\Omega>$ $\max \left(\omega_{\mathrm{A} s}, \omega_{\mathrm{A} z}\right)$, then we recover the instability condition for non-magnetic stars (see Eq. (23) of the paper by Urpin 2003). Assuming $\omega_{\mathrm{A} s} \sim \omega_{\mathrm{A} z} \sim \omega_{\mathrm{A}}=k B / \sqrt{4 \pi \rho}$, we obtain that the non-magnetic criterion applies if

$\lambda>\frac{P B}{\sqrt{4 \pi \rho}}=2.8 \times 10^{5} B_{13} P \rho_{14}^{-1 / 2} \mathrm{~cm}$.

For example, if $B \sim 10^{13} \mathrm{G}$ and $P \sim 10 \mathrm{~ms}$ then the nonmagnetic criterion applies for perturbations with the wavelength $\lambda>30 \mathrm{~m}$.

If the star rotates slowly, $\omega_{\mathrm{BV}} \gg \Omega$ (and $\alpha_{1} \gg 1$ ), but the magnetic field is strong, $\omega_{\mathrm{A}} \gg \Omega$, then the condition of instability is

$\left(\frac{\partial \Omega}{\partial z}\right)^{2}>\frac{\alpha_{1}}{s^{2}}\left(\omega_{\mathrm{A} s} \sin \theta+\omega_{\mathrm{A} z} \cos \theta\right)^{2}$. 
This condition is much more restricting than the corresponding criterion for non-magnetic stars. Estimating $|\partial \Omega / \partial z| \sim \Delta \Omega / s$, we obtain from Eq. (33)

$\frac{\Delta \Omega}{\Omega}>\frac{\mu}{\sqrt{2}} \cdot \frac{\omega_{\mathrm{BV}}}{\Omega} \cdot \frac{\omega_{\mathrm{A}}}{\Omega}$.

Since $\omega_{\mathrm{BV}} \gg \Omega$ and $\omega_{\mathrm{A}} \gg \Omega$, the condition (34) (and, hence, the condition (21)) likely does not fulfil in slowly rotating magnetic neutron stars.

If the star rotates rapidly, $\Omega \gg \omega_{\mathrm{BV}}\left(\alpha_{1} \ll 1\right)$, then the condition of instability in a strong field with $\omega_{\mathrm{A}}>\Omega$ is

$\left(\frac{\partial \Omega}{\partial z}\right)^{2}>\frac{1}{s^{2} \Omega^{2}}\left(\Omega_{\mathrm{e}}^{2} \omega_{\mathrm{A} s}^{2}+2 \Omega s \frac{\partial \Omega}{\partial z} \omega_{\mathrm{A} s} \omega_{\mathrm{A} z}\right)$.

This inequality can be satisfied if

$\frac{\partial \Omega}{\partial z}>\frac{1}{s \Omega}\left[\omega_{\mathrm{A} s} \omega_{\mathrm{A} z}+\sqrt{\omega_{\mathrm{A} s}^{2}\left(\omega_{\mathrm{A} z}^{2}+\Omega_{\mathrm{e}}^{2}\right)}\right]$,

or

$\frac{\partial \Omega}{\partial z}<\frac{1}{s \Omega}\left[\omega_{\mathrm{A} s} \omega_{\mathrm{A} z}-\sqrt{\omega_{\mathrm{A} s}^{2}\left(\omega_{\mathrm{A} z}^{2}+\Omega_{\mathrm{e}}^{2}\right)}\right]$.

Assuming $\omega_{\mathrm{A} s} \sim \omega_{\mathrm{A} z} \sim \omega_{\mathrm{A}}$, we can estimate that these inequalities apply if

$\frac{\Delta \Omega}{\Omega}>\left(\frac{\omega_{\mathrm{A}}}{\Omega}\right)^{2} \gg 1 \quad$ or $\quad \frac{\Delta \Omega}{\Omega}<-1$.

In both cases, to set instability one needs a stronger vertical gradient of $\Omega$ (positive or negative) than in non-magnetic stars. Therefore, we have to conclude that the instability associated with the condition $A_{1}<0$ can likely manifest itself only for perturbations with the wavelength satisfying the inequality (32).

\section{The condition $A_{2}<0$}

The condition $A_{2}<0$ is equivalent to

$$
\begin{aligned}
& {\left[\left(\omega_{\chi}+\omega_{v}\right) \omega_{\mathrm{g}}^{2}+2 \omega_{\nu} Q^{2}\right]\left(\omega_{\chi} Q^{2}+\omega_{\nu} \omega_{\mathrm{g}}^{2}\right)+\omega_{\chi}^{2} \omega_{\mathrm{A}}^{2}} \\
& \quad \times\left[\omega_{\mathrm{g}}^{2}+2 \eta\left(4 \Omega^{2} \frac{k_{z}^{2}}{k^{2}}+\omega_{\mathrm{A}}^{2}+2 Q^{2}\right)+16 \eta^{2} \Omega^{2} \frac{k_{z}^{2}}{k^{2}}\right]<0,
\end{aligned}
$$

where $\eta=v / \chi$. If $v=0$ and stratification is stable, this inequality recovers the well-known criterion of the Goldreich-Schubert instability, modified by the magnetic field,

$Q^{2}+\omega_{\mathrm{A}}^{2}<0$.

If $B=0$, then we have from Eq. (39)

$\left[\left(\omega_{\chi}+\omega_{\nu}\right) \omega_{\mathrm{g}}^{2}+2 \omega_{\nu} Q^{2}\right]\left(\omega_{\chi} Q^{2}+\omega_{\nu} \omega_{\mathrm{g}}^{2}\right)<0$.

This criterion can be fulfilled if either

$\left(\omega_{\chi}+\omega_{\nu}\right) \omega_{\mathrm{g}}^{2}+2 \omega_{\nu} Q^{2}<0$,

or

$\omega_{\chi} Q^{2}+\omega_{\nu} \omega_{\mathrm{g}}^{2}<0$.
The first inequality is a non-magnetic limit of the condition (19), and the second inequality represent the criterion of instability of non-magnetic stars considered by Urpin (2003).

If the Rayleigh stability criterion is fulfilled, the condition (39) can be satisfied only at $\partial \Omega / \partial z \neq 0$. Generally, the presence of the magnetic field stabilizes differential rotation in the same manner as it does in the criterion (19). In a sufficiently strong field with $\omega_{\mathrm{A}} \gg \max \left(\omega_{\mathrm{g}}, \Omega\right)$, one needs a very large $z$-gradient of $\Omega$ to satisfy Eq. (39),

$\left|\frac{\partial \Omega}{\partial z}\right| \geq \frac{1}{s \Omega} \omega_{\mathrm{A} s} \omega_{\mathrm{A} z} \gg \frac{\Omega}{s}$.

If the magnetic field is weak in the sence that $\omega_{\mathrm{A}}<\min \left(\omega_{\mathrm{g}}, \Omega\right)$ but rotation is fast ( $\Omega \gg \omega_{\mathrm{BV}}$ ), then the inequality (39) can be satisfied if

$\left|\frac{\partial \Omega}{\partial z}\right|>\alpha_{2}^{1 / 2}|\cos \theta| \frac{\sqrt{\Omega_{\mathrm{e}}^{2}}}{s}$,

where $\alpha_{2}=\eta\left(\omega_{\mathrm{BV}} / \Omega\right)^{2}$. If we estimate $|\partial \Omega / \partial z| \sim \Delta \Omega / s$ and $\Omega_{\mathrm{e}} \sim \Omega$ then the inequality (43) applies at

$\Delta \Omega>\eta^{1 / 2} \omega_{\mathrm{BV}}$.

Since $\eta \sim 1$ and $\omega_{\mathrm{BV}} \sim 10^{2}-10^{3} \mathrm{~s}^{-1}$, the instability can occur even in stars with a relatively weak differential rotation, $\Delta \Omega \geq \omega_{\mathrm{BV}} \sim 10^{2}-10^{3} \mathrm{~s}^{-1}$. Note that $\omega_{\mathrm{BV}}$ decreases when the neutron star cools down and, hence, older neutron stars require a smaller vertical gradient of the angular velocity to set instability.

If rotation is slow and $\omega_{\mathrm{BV}} \gg \Omega$ then the instability arises if differential rotation is strong with either

$|\cos \theta \sin \theta| \frac{\partial \Omega}{\partial z}>\cos ^{2} \theta \frac{\Omega_{\mathrm{e}}^{2}}{2 s \Omega}$,

or

$\frac{\partial \Omega}{\partial z}<-\frac{\alpha_{2} \Omega}{s}|\sin \theta \cos \theta|$.

The condition (47) requires $\Delta \Omega \sim \Omega$, at least (except the region near the equator), whereas the condition (48) applies at an extremely large negative gradient of $\Omega$.

Note that the condition (39) can be very much weakened if the inequality (19) is fulfilled. Of course, an additional criterion has no impact on the principal conclusion concerning stability in this case. However, if both criteria (19) and (39) are satisfied then the number of unstable modes can be larger in accordance with the Routh theorem (see, e.g., DiStefano III et al. 1994).

\section{The condition $A_{3}<0$}

In general, the condition $A_{3}<0$ is too cumbersome for analysis. Some important qualitative conclusions, however, can be obtained for this condition in the limiting cases of strong and weak magnetic fields.

In a strong magnetic field with $\left|\omega_{\mathrm{A}}\right| \gg \max \left(\omega_{\mathrm{g}}, \Omega\right)$, the inequality $A_{3}<0$ can be fulfilled only in the region where the gradient of $\Omega$ is extremely large,

$|\nabla \Omega| \gg \frac{\left|\omega_{\mathrm{A}}^{2}\right|}{s \Omega}$. 
Unlikely that such a large gradient exists in neutron stars and, therefore, perturbations with $\left|\omega_{\mathrm{A}}\right| \gg \max \left(\omega_{\mathrm{g}}, \Omega\right)$ are probably stable.

In the case of a weak magnetic field with $\left|\omega_{\mathrm{A}}\right| \ll \Omega$ or, that is the same, with $\lambda$ stisfying the condition (32), the instability condition requires again a very large $|\nabla \Omega|$ if rotation is slow, $\omega_{\mathrm{BV}}>\Omega$. In rapidly rotating stars with $|\boldsymbol{r} \cdot \nabla \Omega| \gg \omega_{\mathrm{BV}}$, however, the situation seems to be more favorable. The condition of instability reads

$Q^{2}\left[2 \eta(1+2 \eta) Q^{2}+8\left(1+\eta-2 \eta^{2}\right) \Omega^{2} \frac{k_{z}^{2}}{k^{2}}\right]<0$.

This inequality can be fulfilled if either

$Q^{2}<0$

or

$Q^{2}<-\frac{4\left(1+\eta-2 \eta^{2}\right)}{\eta(1+2 \eta)} \frac{k_{z}^{2}}{k^{2}} \Omega^{2}$.

If both Eqs. (51) and (52) are satisfied then $A_{3}>0$. The condition (51) is that of the Goldreich-Schubert instability that occurs if the angular velocity depends on the $z$-coordinate. If $\Omega_{\mathrm{e}}^{2}>0$ then the condition (51) can be satisfied by a proper choice of the wavevector (see Eq. (18)). The condition (52) can even be less restricting if $\eta>1$. In this case, the right hand side is positive, and the range of unstable wavevectors is wider. Note, however, that both Eqs. (51) and (52) have been derived under the assumptions $\Omega>\omega_{\mathrm{BV}}$ and $|\boldsymbol{r} \cdot \nabla \Omega|>\omega_{\mathrm{BV}}$ that implies a fast differential rotation.

\section{Conclusion}

We have considered the stability properties of differentially rotating magnetic neutron stars. Differential rotation can be caused either by core collapse or the merger of a binary neutron star. Numerical simulations indicate indeed that the rotation law can be complex in newly formed neutron stars. The characteristic timescale of viscous dissipation of differential rotation is rather long and, therefore, these stars can be subject to various hydromagnetic instabilities.

The presence of the magnetic field can essentially influence the stability properties of neutron stars. The origin of the magnetic field in neutron stars is still debatable but it cannot be excluded that the field exists in the core during the early evolutionary stage. For example, the field in a collapsing star can be amplified by many orders of magnitude because of the conservation of the magnetic flux. At the end of core collapse, the protoneutron star may have a poloidal magnetic field $\sim 10^{12} \mathrm{G}$, comparable to that observed in young radio pulsars. The toroidal field, however, might be even stronger (Ardelyan et al. 1980). The magnetic field in the core can also be generated by turbulent dynamo mechanism since protoneutron stars are convectively unstable at the beginning of their evolution. The most optimistic estimates lead some authors (Thompson \& Duncan 1993; Thompson \& Murray 2001) to the conclusion that the turbulent dynamo could generate magnetic fields as strong as $10^{15}-10^{16} \mathrm{G}$. Certainly, such fields could influence the stability properties of differentially rotating neutron stars.

In non-magnetic neutron stars, differential rotation satisfying the Rayleigh stability criterion can be unstable only if the angular velocity depends on the $z$-coordinate (Urpin 2003). Contrary to ordinary stars where instability arises at any $\partial \Omega / \partial z \neq 0$, the rotational instability can appear in neutron stars only if $|\partial \Omega / \partial z|$ exceeds some threshold value. This value depends on the the angular velocity as well as on the kinetic coefficients, and can be small for rapidly rotating stars with $\Omega>\omega_{\mathrm{BV}}$ or large for slowly rotating stars with $\Omega<\omega_{\mathrm{BV}}$. The difference to ordinary stars is caused by a particular character of kinetic processes in neutron stars where viscosity is large and generally comparable to the thermal diffusivity. The critical value of the period, discriminating between the rapid unstable and slow stable rotation in non-magnetic neutron stars, lies around $0.1-0.01 \mathrm{~s}$.

The situation can be essentially different if there is the magnetic field in the neutron star core. It is well known that the magnetic field may stabilize a fluid against instabilities. On the other hand, the field can lead to new branches of instability associated, for instance, with Alfvén waves. In magnetic neutron stars, one of these new branches represented by the criterion (12) can be unstable even at a relatively weak differential rotation. The criterion (12) requires either $\partial \Omega \partial s<0$ or $\partial \Omega / \partial z \neq 0$ for instability. The condition (12) can be fulfilled for both rapidly and slowly rotating neutron stars if the magnetic field in the core is not too strong. This conclusion is of principal difference to non-magnetic stars where only rapid differential rotation can be unstable. Note that instabilities represented by other criteria can operate in rapidly rotating magnetic neutron stars as well, but probably they are suppressed in slowly rotating magnetic stars.

The number of modes which are unstable may vary depending on the rotational law. This number is determined by Routh criterium (DiStefano III et al. 1994). For Eq. (8) with $a_{4}>0$, the number of unstable modes is given by the number of changes of sign in the sequence

$$
\left\{1, A_{1}, \frac{A_{2}}{A_{1}}, \frac{A_{3}}{A_{2}}, a_{0}\right\} \text {. }
$$

For example, if only the criterion $a_{0}<0$ is fulfilled then one real mode is unstable. Since $a_{0}$ is proportional to $\chi$ instability associated with the condition (12) turns out to be secular rather than dynamical. Therefore, this unstable mode in magnetic neutron stars grows on the thermal time scale that is much longer compared to the period of rotation. If some other criteria (9) are also fulfilled then the number of unstable modes can be larger.

A stabilizing influence of the magnetic field is of particular importance for perturbations with a short wavelength. For instance, the standard pulsar magnetic field $\sim 10^{12}-10^{13} \mathrm{G}$ can provide a stabilizing effect only for perturbations with $\lambda<$ $10 \mathrm{~m}$ whereas perturbations with longer $\lambda$ will be unstable. Note that the field with the strength $\sim 10^{15}-10^{16} \mathrm{G}$ that is believed to be typical for magnetars can stabilize any differential rotation. 
In the present paper, we have addressed the behaviour of only axisymmetric short wavelength perturbations. It is quite probable, however, that the considered instabilities can operate on larger scales and for non-axisymmetric perturbations. We will address this problem elsewhere. Interaction of the neutron star crust with turbulent hydrodynamic motions caused by considered instabilities may result in small irregularities in the measured periods of young pulsars. These irregularities should be more pronounced in rapidly rotating stars.

Acknowledgements. This work was supported by the grant of Spanish Ministery of Science and Technology (AYA2001-3490-C02-02).

\section{References}

Acheson, D. J. 1978, Phil. Trans. Roy. Soc. Lond. A, 289, 459 Acheson, D. J. 1979, Sol. Phys., 62, 23

Aleksandrov, A., Kolmogorov, A., \& Laurentiev, M. 1985, Mathematics: Its Content, Methods, and Meaning, MIT, Cambridge

Ardelyan, N., Bisnovatyi-Kogan, G., \& Popov, Y. 1980, Sov. Astron., 23, 705

Balbus, S. 1995, ApJ, 453, 380

Baumgarte, T., Shapiro, S., \& Shibata, M. 2000, ApJ, 528, L29

Chandrasekhar, S. 1960, Proc. Natl. Acad. Sci., 46, 253
Chandrasekhar, S. 1961, Hydrodynamic and Hydromagnetic Stability (Oxford: Clarendon)

Cutler, C., Lindblom, L., \& Splinter, R. 1990, ApJ, 230, 847

Dimmelmeier, H., Font, J. A., \& Müller, E. 2002, A\&A, 393, 523

Fricke, K. 1969, A\&A, 1, 388

Kitchatinov, L., \& Rüdiger, G. 1997, MNRAS, 286, 757

Landau, L. D., \& Lifshitz, E. M. 1981, Fluid Mechanics (London: Pergamon)

Maxwell, O. V. 1979, ApJ, 231, 201

Miralles, J. A., Pons, J. A., \& Urpin, V. 2000, ApJ, 543, 1001

Miralles, J. A., Pons, J. A., \& Urpin, V. 2002, ApJ, 574, 356

Rampp, M., Müller, E., \& Ruffert, M. 1998, A\&A, 332, 969

Rasio, F., \& Shapiro, S. 1994, ApJ, 432, 242

Rezzolla, L., Lamb, F., \& Shapiro, S. 2000, ApJ, 531, L141

Shapiro, S. 2000, ApJ, 544, 397

Shapiro, S., \& Teukolsky, S. 1983, Black Holes, White Dwarfs, and Neutron Stars (New York: Wiley)

Shibata, M., \& Uryu, K. 2000, Phys. Rev. D, 61, 064001

Spruit, H. 1999, A\&A, 341, L1

Thompson, C., \& Duncan, R. 1993, ApJ, 408, 194

Thorsen, S., Arzoumanian, Z., McKinnon, M., \& Taylor, J. 1993, ApJ, 405, 129

Thompson, C., \& Murray, N. 2001, ApJ, 560, 339

Urpin, V. 1996, MNRAS, 280, 149

Urpin, V. 2003, A\&A, submitted

Velikhov, E. P. 1959, Sov. Phys. JETP, 9, 995

Zwerger, T., \& Müller, E. 1997, A\&A, 320, 209 